\title{
Quantification of quantum correlation of ensemble of states
}

\author{
Michał Horodecki, Aditi Sen(De), and Ujjwal Sen \\ Institute of Theoretical Physics and Astrophysics, University of Gdańsk, 80-952 Gdańsk, Poland
}

\begin{abstract}
We present first measure of quantum correlation of an ensemble of multiparty states. It is based on the idea of minimal entropy production in a locally distinguishable basis measurement. It is shown to be a relative entropy distance from a set of ensembles. For bipartite ensembles, which span the whole bipartite Hilbert space, the measure is bounded below by average relative entropy of entanglement. We naturally obtain a monotonicity axiom for any measure of quantum correlation of ensembles. We evaluate this measure for certain cases. Subsequently we use this measure to propose a complementarity relation between our measure and the accessible information obtainable about the ensemble under local operations. The measure along with the monotonicity axiom are well-defined even for the case of a single system, where the complementarity relation is seen to be yet another face of the "Heisenberg uncertainty relation".
\end{abstract}

Quantifying quantum correlation of states is a deeply studied problem [1, 2, 3, 4, 5] in quantum information. Several measures has been proposed. Prominent among them are distillable entanglement [1, 6], entanglement of formation [1], relative entropy of entanglement 2], etc.

However to our knowledge, the problem of quantification of quantum correlation of an ensemble of states has never been studied. In this paper we propose first measure of quantum correlation $(\mathcal{Q})$ of an ensemble of states. A trivial way to quantify quantum correlation of an ensemble of states is to take an average of an individual property of the constituent states in the ensemble. E.g., one could take the average entanglement of formation 1] of the ensemble states. However such an averaging over a property of the individual states cannot capture the complexity of the ensemble as a whole. To take into account the structure of the ensemble as a whole, the measure must depend on the ensemble as a whole. A simple measure of quantum correlation of multiparty ensembles could be the difference $\mathbb{D}$ between the globally accessible information and the locally accessible information. However accessible information is an operationally useful quantity, and one aim of defining $\mathcal{Q}$ is to estimate accessible information under different sets of operations. Moreover $\mathbb{D}$ vanishes for single-party ensembles, whereas $\mathcal{Q}$ will be seen to be nontrivial also in such cases.

After defining the measure, we show that it can be seen as a relative entropy distance from a set of "classical" ensembles. For a bipartite ensemble, which spans the whole bipartite Hilbert space, we show that measure is bounded below by the average relative entropy of entanglement [2]. In discussing some of its properties, we naturally obtain a monotonicity axiom for any measure of quantum correlation of ensembles. In a natural way, this gives us the first axiom for any measure of quantumness of an ensemble of a single system. We then evaluate the measure for the case of four Bell states (given with equal prior probabilites). We also evaluate the measure for other more general ensembles. We subsequently propose a complementarity relation [7, 8] between this measure of quantum correlation of an ensemble and the accessible information obtainable about the ensemble, under the relevant class of allowable operations. In the case of a single system, this complementarity is partially proven, and is seen to be yet another face of the "Heisenberg uncertainty relation". Importantly, both the information gathering and disturbance terms in this relation are information-theoretic, in contrast to e.g. 9].

Consider an ensemble of bipartite states $\mathcal{E}=$ $\left\{p_{x},\left|\psi_{x}\right\rangle^{A B}\right\}_{x=1}^{N}$, that are not necessarily orthogonal. One can for example think of a source that produces the state $\left|\psi_{x}\right\rangle^{A B}$ (belonging to the Hilbert space $\mathcal{H}_{A} \otimes \mathcal{H}_{B}$ ) with probability $p_{x}(x=1,2, \ldots, N)$, and sends it to two far apart parties Alice(A) and Bob(B). Here of course the probabilities $p_{x}$ are nonnegative and sum up to unity. Let $\mathcal{A}$ be the span of the states $\left|\psi_{x}\right\rangle^{A B}$. Let $\left\{\left|a_{i}\right\rangle^{A B}\right\}_{i=1}^{n}$ be an orthonormal basis of $\mathcal{H}_{A} \otimes \mathcal{H}_{B}$. Consider the part of $\left\{\left|a_{i}\right\rangle^{A B}\right\}_{i=1}^{n}$ which has a nonzero overlap with $\mathcal{A}[10]$. This part (with nonzero overlap with $\mathcal{A}$ ) can be distinguishable or indistinguishable under local operations and classical communication (LOCC). Let $\mathcal{D}_{\mathcal{A}}$ be the set of all such bases $\left\{\left|a_{i}\right\rangle^{A B}\right\}_{i=1}^{n}$ whose overlap with $\mathcal{A}$ is LOCCdistinguishable. Of course, $n=\operatorname{dim}\left\{\mathcal{H}_{A} \otimes \mathcal{H}_{B}\right\} \leq N$.

The measure of quantum correlation $\mathcal{Q}$ for the ensemble $\mathcal{E}$ is defined as the minimum entropy produced when dephased (measured) in a basis from $\mathcal{D}_{\mathcal{A}}$. More precisely,

$$
\mathcal{Q}(\mathcal{E})=\min _{\mathcal{D}_{\mathcal{A}}} \sum_{x=1}^{N} p_{x} S\left(\sum_{i=1}^{n} p_{i \mid x}\left|a_{i}\right\rangle\left\langle a_{i}\right|\right) .
$$

Here $p_{i \mid x}$ is the probability that $\left|a_{i}\right\rangle$ clicks if the signal state was $\left|\psi_{x}\right\rangle$, i.e. $p_{i \mid x}=\left|\left\langle\psi_{x} \mid a_{i}\right\rangle\right|^{2}$. $S$ denotes the von Neumann entropy, defined as $S(\eta)=-\operatorname{tr} \eta \log _{2} \eta$. Since $\left|a_{i}\right\rangle$ are orthonormal, $S\left(\sum_{i=1}^{n} p_{i \mid x}\left|a_{i}\right\rangle\left\langle a_{i}\right|\right)=$ $H\left(\left\{p_{i \mid x}\right\}_{i=1}^{n}\right)$. Here $H$ denotes the Shannon entropy, defined as $H\left(\left\{q_{j}\right\}_{j=1}^{m}\right)=-\sum_{j=1}^{m} q_{j} \log _{2} q_{j}$, where $\left\{q_{j}\right\}_{j=1}^{m}$ forms a set of probabilities. Therefore

$$
\mathcal{Q}(\mathcal{E})=\min _{\mathcal{D}_{\mathcal{A}}} \sum_{x=1}^{N} p_{x} H\left(\left\{p_{i \mid x}\right\}_{i=1}^{n}\right) .
$$

The minimum is taken over all bases in $\mathcal{D}_{\mathcal{A}}$. 
It is interesting to note that one may define $\mathcal{Q}$, for the ensemble $\mathcal{E}$, as the minimum entropy produced when dephased in an arbitrary basis in $\mathcal{H}_{A} \otimes \mathcal{H}_{B}$ so that the resulting ensemble after the measurement is an LOCCdistinguishable set of states.

It is quite straightforward to carry over the above definition to the case of mixed ensembles. For the case of mixed states, one must include in the definition, a subtraction of the initial von Neumann entropy of the signal states. More precisely, consider an ensemble $\mathcal{E}^{\prime}=$ $\left\{p_{x}, \varrho_{x}^{A B}\right\}_{x=1}^{N}$ of bipartite signal states, which are not necessarily pure. In this case, $\mathcal{A}$ will denote the union of the supports of $\varrho_{x}^{A B}$ for $x=1,2, \ldots, N$. The definition of $\mathcal{D}_{\mathcal{A}}$ is then exactly the same as for pure signals. Then

$$
\mathcal{Q}\left(\mathcal{E}^{\prime}\right)=\min _{\mathcal{D}_{\mathcal{A}}} \sum_{x=1}^{N} p_{x}\left(H\left(\left\{p_{i \mid x}\right\}_{i=1}^{n}\right)-S\left(\varrho_{x}\right)\right),
$$

where $p_{i \mid x}=\left\langle a_{i}\left|\varrho_{x}^{A B}\right| a_{i}\right\rangle$.

Actually, the above notion of $\mathcal{Q}$ as a measure of quantumness can be used in much more general situations than just in the case of a system consisting of two spatially localized subsystems. Suppose that a source produces an ensemble $\left\{p_{x}, \varrho_{x}\right\}_{x=1}^{N}$, with the $\varrho_{x}$ 's defined on some Hilbert space $\mathcal{H}$. And as before, let $\mathcal{A}$ denote the union of the supports of $\varrho_{x}$, and let $\left\{\left|a_{i}\right\rangle\right\}_{i=1}^{n}$ be an arbitrary complete basis in $\mathcal{H}$. Depending on a set of allowed operations, say $\Lambda$, the nonzero overlap [10] of $\left\{\left|a_{i}\right\rangle\right\}_{i=1}^{n}$ with $\mathcal{A}$ may or may not be distinguishable. Let $\mathcal{D}_{\mathcal{A}}^{\Lambda}$ be the set of all bases $\left\{\left|a_{i}\right\rangle\right\}_{i=1}^{n}$ for which the nonzero overlap with $\mathcal{A}$ is distinguishable under $\Lambda$. Then the measure of quantumness of the ensemble $\Gamma=\left\{p_{x}, \varrho_{x}\right\}_{x=1}^{N}$, with respect to the set of allowed operations $\Lambda$, is defined as

$$
\mathcal{Q}(\Gamma)=\min _{\mathcal{D}_{\mathcal{A}}^{\Lambda}} \sum_{x=1}^{N} p_{x}\left(H\left(\left\{p_{i \mid x}\right\}_{i=1}^{n}\right)-S\left(\varrho_{x}\right)\right),
$$

where $p_{i \mid x}$ is, as before, the probability that $\left|a_{i}\right\rangle$ clicks given that the signal was $\varrho_{x}$.

When the states $\varrho_{x}$ are bipartite states between A and $\mathrm{B}$, with the Hilbert space $\mathcal{H}$ split as $\mathcal{H}_{A} \otimes \mathcal{H}_{B}$, and with the allowed operations being LOCC between A and B, we recover the definition given in eq. (1).

For a multiparty ensemble $\left\{p_{x}, \varrho_{x}^{A B C \ldots}\right\}$, defined on the Hilbert space $\mathcal{H}_{A} \otimes \mathcal{H}_{B} \otimes \mathcal{H}_{C} \otimes \ldots$, one chooses the bases $\left\{\left|a_{i}\right\rangle^{A B C \cdots}\right\}_{i=1}^{n}$ spanning this Hilbert space. Then considering the set of allowed operations as LOCC between $\mathrm{A}, \mathrm{B}, \mathrm{C}, \ldots, \mathcal{D}_{\mathcal{A}}$ is the set of bases whose nonzero overlap with $\mathcal{A}$ is distinguishable by such LOCC operations, where $\mathcal{A}$ is the union of the supports of $\varrho_{x}^{A B C \cdots}$. In this way, we have a measure of quantum correlation of an ensemble of multiparty states.

Importantly, $\mathcal{Q}$ can be defined also for ensembles of single systems. Then $\mathcal{Q}$ is the quantity in eq. (2), with the allowed operations being all quantum mechanical operations. Consequently the dephasing is in any orthonormal basis in $\mathcal{H}$. This quantity can be interpreted as a measure of quantumness of the ensemble $\Gamma$.

We now show that $\mathcal{Q}(\Gamma)$ is average relative entropy distance from some ensembles of states. We have (see eq. (2) $\mathcal{Q}(\Gamma)=\min _{\left\{\varsigma_{x}^{\prime}\right\}} \sum_{x} p_{x}\left[-\operatorname{tr}\left(\varrho_{x} \log _{2} \varsigma_{x}^{\prime}\right)+\right.$ $\left.\operatorname{tr}\left(\varrho_{x} \log _{2} \varrho_{x}\right)\right]=\min _{\left\{\varsigma_{x}^{\prime}\right\}} \sum_{x} p_{x} S\left(\varrho_{x} \mid \varsigma_{x}^{\prime}\right)$, so that

$$
\mathcal{Q}(\Gamma)=\min _{\left\{\varsigma_{x}\right\}} \sum_{x} p_{x} S\left(\varrho_{x} \mid \varsigma_{x}\right),
$$

where $\varsigma_{x}^{\prime}=\sum_{i} p_{i \mid x}\left|a_{i}\right\rangle\left\langle a_{i}\left|, \varsigma_{x}=\sum_{i} a_{i}^{x}\right| a_{i}\right\rangle\left\langle a_{i}\right|,\left\{\left|a_{i}\right\rangle\right\}_{i}$ being any set of states which are distinguishable under the set of allowable operations $\Lambda,\left\{a_{i}^{x}\right\}_{i}$ 's being arbitrary sets of probabilities, mixing the $\left|a_{i}\right\rangle$ 's. $S(\varrho \mid \varsigma)=$ $\operatorname{tr}\left(\varrho \log _{2} \varrho-\varrho \log _{2} \varsigma\right)$ is the relative entropy distance of $\varrho$ from $\varsigma$. So $\mathcal{Q}$ is the average relative entropy distance of the signal states from the "classical" ensembles, namely the ones whose members are mixtures of distinguishable states (under $\Lambda$ ). Note that for a single "classical" ensemble, the constituent signals are mutually commuting. Although $\mathcal{Q}$ turns out to be a relative entropy distance, it has (in contrast to relative entropy of entanglement) an operational meaning in terms of entropy production (by which it is defined in eq. (10) even for a single copy of the ensemble. Therefore $\mathcal{Q}$ is more intimately related to quantum correlation measure of states of 11] than to relative entropy of entanglement. Note that the averaging in eq. (3) is done before the minimization, and hence the measure $\mathcal{Q}$ can still "feel" the ensemble as a whole.

Consider now the case of an ensemble of bipartite states with the allowed operations being LOCC between the sharing partners. Suppose also that the union of the supports of the ensemble covers the whole bipartite Hilbert space. Then a set of orthogonal states in the support (i.e. a complete orthogonal basis) is distinguishable under LOCC only if they are all product states [12]. (Note that the opposite is not necessarily true [13].) Ensembles $\left\{\varsigma_{x}\right\}$ that are mixtures of states from the sets $\left\{\left|a_{i}\right\rangle\right\}$ which are distinguishable under LOCC, but do not span the complete bipartite Hilbert space, will (in this case) produce infinite relative entropy distance, and can therefore be ignored (cf. eq. (31)). So the relavant ensembles that appear in formula (3) are all separable states. (Again the opposite is not true.) Thus we have

$$
\mathcal{Q}\left(\mathcal{E}^{\prime}\right) \geq \overline{E_{R}}=\sum_{x} p_{x} E_{R}\left(\varrho_{x}^{A B}\right),
$$

for the ensemble $\mathcal{E}^{\prime}=\left\{p_{x}, \varrho_{x}^{A B}\right\}$, where we have assumed that the union of the supports of the $\varrho_{x}^{A B}$ 's cover the whole bipartite Hilbert space. $E_{R}$ is the relative entropy of entanglement defined as $E_{R}\left(\varrho^{A B}\right)=\min _{\varsigma} S(\varrho \mid \varsigma)$, where the minimization is over all separable states $\varsigma$ [2]. Among other things, the inequality (4) will be important for evaluation of $\mathcal{Q}$ for certain bipartite ensembles.

As $\mathcal{Q}$ is defined in terms of von Neumann entropy, it will inherit some continuity properties due to the Fannes' 
inequality [14]. By definition, $\mathcal{Q}$ is vanishing for ensembles which are distinguishable under the allowed set of operations $\Lambda$. And it is nonzero otherwise.

It should be noted that $\mathcal{Q}$, for the case of multipartite ensembles (with the allowed operations being the LOCC class) can actually increase under LOCC. This is because one can create nonorthogonal product states after starting with a multi-orthogonal product basis 15]. Note however that $\mathcal{Q}$ can increase even if the output states are orthogonal, as there exist ensembles of orthogonal product states which are locally indistinguishable 13]. Such ensembles, which by definition has nonzero $\mathcal{Q}$, can be created by LOCC from a multi-orthogonal product basis [15], which being LOCC distinguishable has by definition vanishing $\mathcal{Q}$. However, from our experience with entanglement-like quantum correlation measures [1, 2, 3, 4, 5], we know that a quantum correlation measure should show some kind of monotonicity. We believe that $\mathcal{Q}$, for the case of multipartite ensembles, will be monotonically decreasing under LOCC operations, if we do not allow tracing out as a valid operation. Note that tracing out can never be useful in a distinguishing protocol. The accessible information under LOCC, for an ensemble of multipartite states, is the same as the accessible information under LOCC without tracing out as a valid operation. The operations that can be useful in attaining the accessible information are (a) adding local ancillas, (b) local unitarities, (c) local dephasing (von Neumann measurement and then forgetting the outcome), (d) communication of pre-dephased quantum states (classical communication). Adding the item (e) tracing out, will give us the whole set of LOCC. $\mathcal{Q}$ is obviously monotonically decreasing for operations (a), (b), and (d). We conjecture that $\mathcal{Q}$ is nonincreasing also for local dephasing of multipartite ensembles. Therefore we have a natural axiom for any measure of quantum correlation of ensembles of multipartite states, viz. it must be nonincreasing under (a), (b), (c), and (d). Let us emphasize that the axiom holds for measures of multipartite ensembles, not only for bipartite ones. Moreover, nonincreasingness under addition of ancillas, unitary operations, and dephasing is in this way seen to be a natural axiom for any measure of quantumness of a single system.

We will now calculate the value of our measure for the ensemble $\mathcal{B}$ consisting of the four Bell states, $\left|\phi^{ \pm}\right\rangle=$ $\frac{1}{\sqrt{2}}(|00\rangle \pm|11\rangle),\left|\psi^{ \pm}\right\rangle=\frac{1}{\sqrt{2}}(|01\rangle \pm|10\rangle)$, given with equal prior probabilities. The four Bell states span the whole $2 \otimes 2$ Hilbert space. To find $\mathcal{Q}$, we have to minimize entropy production after dephasing over all LOCCdistinguishable bases in $2 \otimes 2$. Let us first dephase in the computational basis $\{|00\rangle,|11\rangle,|01\rangle,|10\rangle\}$. Suppose for example, that the signal is $\left|\phi^{+}\right\rangle$. Then either $|00\rangle$ or $|11\rangle$ clicks with equal probabilities. So the entropy produced is $H\left(\frac{1}{2}\right)$, where $H(\cdot)$ is the binary entropy function, defined as $H(p)=-p \log _{2} p-(1-p) \log _{2}(1-p), 0 \leq p \leq 1$.
The signal $\left|\phi^{+}\right\rangle$is created by the source with probability $\frac{1}{4}$. The case is similar for the other signals. Therefore we obtain $\mathcal{Q} \leq 4 \times \frac{1}{4} H\left(\frac{1}{2}\right)=1$. However from ineq. (4) we know that this bound is saturated, as the Bell states have $E_{R}=1$. Therefore, we have that $\mathcal{Q}=1$ for the ensemble of the four Bell states given with equal prior probabilities. Consider now the more general ensemble $\mathcal{B}^{\prime}$ consisting of $a|00\rangle+b|11\rangle, \bar{b}|00\rangle-\bar{a}|11\rangle, c|01\rangle+d|10\rangle, \bar{d}|01\rangle-\bar{c}|10\rangle$, given with equal prior probabilities. Again a dephasing in the computational basis $\{|00\rangle,|11\rangle,|01\rangle,|10\rangle\}$, implies that $\mathcal{Q} \leq \frac{1}{2}\left(H\left(|a|^{2}\right)+H\left(|c|^{2}\right)\right)$. And again saturation follows from ineq. (4i), so that $\mathcal{Q}=\frac{1}{2}\left(H\left(|a|^{2}\right)+H\left(|c|^{2}\right)\right)$. Similar calculations will deliver us also the value of $\mathcal{Q}$ for the canonical set of maximally entangled states in $d \otimes d$, given with equal prior probabilities, viz. $\left|\psi_{n m}^{\max }\right\rangle=$ $\frac{1}{\sqrt{d}} \sum_{j=0}^{d-1} e^{2 \pi i j n / d}|j\rangle|(j+m) \bmod \quad d\rangle(n, m=0, \ldots, d-$ 1). Considering a dephasing of this basis in the computational basis, and using ineq. (4), one obtains $\mathcal{Q}=\log _{2} d$.

Quantification of quantum correlation of an ensemble of states has several important potential applications. E.g., it can be used to obtain a complementarity relation with locally accessible information (accessible information under LOCC-based measurements). For an ensemble $\left\{p_{x}, \varrho_{x}^{A B}\right\}$ of bipartite states, a complementarity has been obtained between the locally accessible information and average shared entanglement of the ensemble states [8]. Precisely, if $I_{a c c}^{L O C C}$ denotes the locally accessible information for the ensemble $\left\{p_{x}, \varrho_{x}^{A B}\right\}$, then one has [8]

$$
I_{a c c}^{L O C C}+\bar{E} \leq \log _{2} n,
$$

where ensemble states $\varrho_{x}^{A B}$ are defined on $\mathcal{H}_{A} \otimes \mathcal{H}_{B}, n=$ $\operatorname{dim}\left\{\mathcal{H}_{A} \otimes \mathcal{H}_{B}\right\}$, and $\bar{E}=\sum_{x} p_{x} E\left(\varrho_{x}^{A B}\right), E$ being any asymptotic entanglement measure of bipartite states [5].

However the complexity of locally accessible information in comparison to globally accessible information depends on the geometry of the ensemble, which cannot be captured by taking an average of an individual property (for example, entanglement) of the ensemble states. Consequently, the complementarity obtained in [8] can potentially be made stronger if the locally accessible information is taken along with a measure of quantum correlation of an ensemble of states.

We conjecture that the following complementarity relation holds between the accessible information, $I_{a c c}^{\Lambda}$, for the set of allowed operations $\Lambda$, and $\mathcal{Q}$, for an ensemble $\left\{p_{x}, \varrho_{x}\right\}_{x=1}^{N}$ :

$$
I_{\text {acc }}^{\Lambda}+\mathcal{Q} \leq \log _{2} N .
$$

Among other things, note also the change in the righthand-side of ineq. (6) with respect to that of ineq. (5). In ineq. (5), the right-hand-side is the logarithm of the dimension of the Hilbert space on which the signals are defined. However in ineq. (6), the right-hand-side is the logarithm of the number of states in the ensemble, which 
can be less than, equal to, or greater than the dimension of the said Hilbert space.

For an ensemble of bipartite states, $\left\{p_{x}, \varrho_{x}^{A B}\right\}_{x=1}^{N}$, the proposed complementarity is

$$
I_{a c c}^{L O C C}+\mathcal{Q} \leq \log _{2} N
$$

Note that due to ineq. (4), the relation (7) will in general be stronger than the one in ineq. (5), if the ensemble consists of $n=\operatorname{dim}\left\{\mathcal{H}_{A} \otimes \mathcal{H}_{B}\right\}$ states that spans $\mathcal{H}_{A} \otimes$ $\mathcal{H}_{B}$, and if we replace $E$ by $E_{R}$. We conjecture that the relation (7) is in general stronger than the one in (5), at least when we replace $E$ by $E_{R}$. In particular, we believe that $\mathcal{Q} \geq \overline{E_{R}}+\log _{2} N-\log _{2} n$.

For the case of the four Bell states, given with equal prior probabilities, $I_{a c c}^{L O C C}=1$. This follows from ineq. (5) and the fact that measuring in the computational basis gives $I_{a c c}^{L O C C} \geq 1$. And for this case, we have proven that $\mathcal{Q}=1$. Therefore we have proven inequality (7) for the case of four Bell states given with equal probabilities. Ineq. (7) is true also for the more general ensemble $\mathcal{B}^{\prime}$. From ineq. (5), we have that for the ensemble $\mathcal{B}^{\prime}$, $I_{a c c}^{L O C C} \leq 2-\frac{1}{2}\left(H\left(|a|^{2}\right)+H\left(|c|^{2}\right)\right)$. But a measurement in the computational basis shows that this bound can be achieved. Using the value of $\mathcal{Q}$ obtained for $\mathcal{B}^{\prime}$, we have the inequality (7) proven for this ensemble. One may similarly prove the inequality (7) for the canonical set of maximally entangled states in $d \otimes d$.

For the case of the ensemble of the three Bell states $\left|\phi^{ \pm}\right\rangle,\left|\psi^{+}\right\rangle$, given with equal prior probabilities, a measurement in the computational basis gives $I_{a c c}^{L O C C} \geq$ $\log _{2} 3-\frac{2}{3}$. And the fact that set $\left\{|00\rangle,|11\rangle,\left|\psi^{+}\right\rangle\right\}$is an LOCC-distinguishable ensemble in the span of $\left|\phi^{ \pm}\right\rangle$, $\left|\psi^{+}\right\rangle$, gives $\mathcal{Q} \leq \frac{2}{3}$. Note here that these two inequalities do not have a contradiction with the proposed ineq. (7).

One may consider ineq. (6) for the case of an ensemble of a single system, with all quantum mechanically allowed operations in $\Lambda$. Then ineq. (6) can be seen as a "Heisenberg uncertainty relation". The accessible information is the maximal "information gain" that is possible about the system. On the other hand, $\mathcal{Q}$ denotes the quantumness of the ensemble, quantifying the resistance to such information gain. In this case of a single system, it is actually possible to prove the ineq. (6) in a restricted case. For an ensemble $\left\{p_{x}, \varrho_{x}\right\}_{x=1}^{N}$, defined on the Hilbert space $\mathcal{H}$, the accessible information, $I_{a c c}=\max _{M}\left(H\left(\left\{p_{x}\right\}_{x=1}^{N}\right)-\sum_{y} r_{y} H\left(\left\{p_{x \mid y}\right\}_{x=1}^{N}\right)\right)$, where a measurement $M=\{y\}$ has been performed, the measurement result $y$ having occured with probability $r_{y}$. $p_{x \mid y}$ is the probability that the signal was $\varrho_{x}$, given that the outcome was $y$. The maximization is carried over all measurements. The quantity within the maximization is the mutual information between the source producing the ensemble $\left\{p_{x}, \varrho_{x}\right\}_{x=1}^{N}$ and the maesurement outcomes. But we know that $H(X)-H(X \mid Y)=H(Y)-H(Y \mid X)$, for random variables $X$ and $Y$. Therefore $H\left(\left\{p_{x}\right\}_{x=1}^{N}\right)-$ $\sum_{y} r_{y} H\left(\left\{p_{x \mid y}\right\}_{x=1}^{N}\right)=H\left(\left\{p_{y}\right\}_{y}\right)-\sum_{x=1}^{N} p_{x} H\left(\left\{p_{y \mid x}\right\}_{y}\right)$. At this point, we restrict ourselves to only projection valued measurements on $\mathcal{H}$. Then we have $I_{a c c} \leq$ $\max _{M} H\left(\left\{p_{y}\right\}_{y}\right)-\mathcal{Q} \leq \log _{2} \operatorname{dim} \mathcal{H}-\mathcal{Q} \leq \log _{2} N-\overline{\mathcal{Q}}$, which is just the inequality in (6).

It is conceivable that the complementarity in ineq. (6) is true only when we consider a certain form of regularisation of the quantity $\mathcal{Q}$. Given an ensemble $\left\{p_{x}, \varrho_{x}\right\}$ and a set of allowed operations $\Lambda$, consider $\mathcal{Q}_{0}$ for an extension $\left\{p_{x}, \varrho_{x} \otimes|0\rangle\langle 0|\right\}$ of the given ensemble, where $|0\rangle$ is any state that is free under $\Lambda$. The regularization is then the minimum of $\mathcal{Q}_{0}$ 's for all possible such extensions. This brings in the possibility of vanishing regularized $\mathcal{Q}$ even for some ensembles that are indistinguishable under $\Lambda$.

This work is supported by EU grants RESQ and QUPRODIS, and by the University of Gdańsk, Grant No. BW/5400-5-0256-3.

[1] C.H. Bennett, D.P. DiVincenzo, J.A. Smolin, and W.K. Wootters, Phys. Rev. A 54, 3824 (1997).

[2] V. Vedral, M.B. Plenio, M.A. Rippin, and P.L. Knight, Phys. Rev. Lett. 78, 2275 (1997). V. Vedral and M.B. Plenio, Phys. Rev. A 57, 1619 (1998).

[3] G. Vidal, J. Mod. Opt. 47, 355 (2000).

[4] M. Horodecki, P. Horodecki, and R. Horodecki, Phys. Rev. Lett. 84, 2014 (2000).

[5] M. Horodecki, QIC, 1, 3 (2001).

[6] C.H. Bennett, G. Brassard, S. Popescu, B. Schumacher, J.A. Smolin, and W.K. Wootters, Phys. Rev. Lett. 76, 722 (1996).

[7] J. Oppenheim, K. Horodecki, M. Horodecki, P. Horodecki, and R. Horodecki, quant-ph/0207025

[8] P. Badzia̧g, M. Horodecki, A. Sen(De), and U. Sen, Phys. Rev. Lett. 91, 117901 (2003).

[9] C.A. Fuchs and A. Peres, quant-ph/9512023 B.-G. Englert, Phys. Rev. Lett. 77, 2154 (1996).

[10] The vector $\left|a_{i_{k}}\right\rangle$ of the basis $\left\{\left|a_{i}\right\rangle^{A B}\right\}_{i=1}^{n}$ has a "nonzero overlap" with $\mathcal{A}$, if $\left\langle a_{i_{k}} \mid \wp\right\rangle \neq 0$, for some vector $|\wp\rangle \in \mathcal{A}$. The set of such $\left|a_{i_{k}}\right\rangle$ from the basis $\left\{\left|a_{i}\right\rangle^{A B}\right\}_{i=1}^{n}$ is called the "overlap of the basis $\left\{\left|a_{i}\right\rangle^{A B}\right\}_{i=1}^{n}$ with $\mathcal{A}$ " or the "part of the basis $\left\{\left|a_{i}\right\rangle^{A B}\right\}_{i=1}^{n}$ having nonzero overlap with $\mathcal{A}$ ".

[11] J. Oppenheim, M. Horodecki, P. Horodecki, and R. Horodecki, Phys. Rev. Lett. 89, 180402 (2002); M. Horodecki, K. Horodecki, P. Horodecki, R. Horodecki, J. Oppenheim, A. Sen(De), and U. Sen, ibid. 90, 100402 (2003).

[12] M. Horodecki, A. Sen(De), U. Sen, and K. Horodecki, Phys. Rev. Lett. 90, 047902 (2003).

[13] C.H. Bennett, D.P. DiVincenzo, C.A. Fuchs, T. Mor, E.M. Rains, P.W. Shor, J.A. Smolin, and W.K. Wootters, Phys. Rev. A 59, 1070 (1999); C.H. Bennett, D.P. DiVincenzo, T. Mor, P.W. Shor, J.A. Smolin, and B.M. Terhal, Phys. Rev. Lett. 82, 5385 (1999).

[14] M. Fannes, Commun. Math. Phys. 31, 291 (1973).

[15] For orthogonal bases $\{|i\rangle\}_{i=1}^{\operatorname{dim}} \mathcal{H}_{k}$ of $\mathcal{H}_{k}(k=A, B, \ldots)$, $\{|i\rangle \otimes|j\rangle \otimes \ldots\}_{i, j, \ldots=1}^{\operatorname{dim}} \mathcal{H}_{A}, \operatorname{dim} \mathcal{H}_{B}, \ldots$ is the corresponding multiorthogonal basis. Such a basis is trivially distinguishable 
by LOCC between $\mathrm{A}, \mathrm{B}, \ldots$... So an ensemble whose states are from such a basis, will have zero $\mathcal{Q}$. 